\title{
Multi-centers clinicopathological prognosis study of inflammatory myofibroblastic tumors of urogenital system in China
}

Bo Chen

Sichuan University West China Hospital

$\mathrm{He} \mathrm{Xu}$

Sichuan University West China Hospital

Dehong Cao

Sichuan University West China Hospital

Jianbing Guo

Sichuan University West China Hospital

Zeyu Chen

Sichuan University West China Hospital

Yin Huang

Sichuan University West China Hospital

Shi Qiu

Sichuan University West China Hospital

Tianhai Lin

Sichuan University West China Hospital

$\mathrm{Na} \mathrm{Ma}$

Sichuan University West China Second University Hospital

Liangren Liu ( $\square$ liuliangren@scu.edu.cn )

West China Hospital of Sichuan University https://orcid.org/0000-0002-2946-0663

Qiang Wei

Sichuan University West China Hospital

\section{Research article}

Keywords: Inflammatory myofibroblastic tumors, Urogenital system, Kidney, Urinary bladder

Posted Date: July 15th, 2019

DOI: https://doi.org/10.21203/rs.2.11394/v1 
License: (c) (i) This work is licensed under a Creative Commons Attribution 4.0 International License. Read Full License 


\section{Abstract}

Purpose: We aimed at investigate a series of inflammatory myofibroblastic tumors (IMTs) of urogenital system in multi-centers in China. Materials and methods: Within the pathologic databases at West China Hospital of Sichuan University and the Third People's Hospital of Chengdu, we identified 33 individuals with IMTs from May 2009 to January 2019 Results: A total of 33 patients with IMTs of urogenital system were identified. The median age of all the individuals was 39.5 years (range 0-74 years). The most common presentations were hematuria (13patients), pain (12 patients), and mass (12 patients). In addition, other clinical symptoms included dysuria, urinary frequency, and fever. Fourteen individuals were diagnosed with anemia, including 10 mild anemia patients and 4 moderate anemia cases. Moreover, neutrophil was the dominant inflammatory cell type. The main positive markers of immunohistochemistry staining included SMA (22/25), MSA (8/9), CD34 (15/17), and CD68 (3/3). Surgery resection was the sole treatment for all the patients. Follow- up was available in 23 patients during a median follow-up period of 78.3 months (range 19.2-118.8 months), and either recurrence or metastasis was detected in any patients except one patients died from retroperitoneum IMTs. Conclusion: This study confirmed that urogenital system IMTs, with potential of recurrence or metastasis, are extremely rare. In summary, although favorable prognosis was observed in IMTs of urogenital system, closely follow-up is essential and needed.

\section{Background}

Inflammatory myofibroblastic tumors (IMTs), a rare entity of spindle cell tumors, composed of myofibroblastic mesenchymal spindle cells with an inflammatory infiltrate of plasma cells, lymphocytes, and eosinophils ${ }^{[1]}$. According to World Health Organization (WHO) classification, IMTs are intermediate tumors with potential for recurrence. The first report of IMTs was demonstrated by Brunn on two cases of "myoma of the lung" in 1939, and IMTs of urinary system was firstly described by Roth in $1980^{[2]}$. The lung is the most common site (95\%) of IMTs, and other potential sites (5\%) including abdomen, retroperitoneum, pelvic cavity, head, neck, trunk, and limbs ${ }^{[3]}$. What's more, urinary bladder is the most common site of IMTs. Nevertheless, IMTs comprise less than $1 \%$ of all bladder neoplasms ${ }^{[4]}$.

Nevertheless, IMTs of urinary system couldn't be recognized precisely because of clinical symptoms, laboratory test results and imaging lacking specificity for the diagnosis of IMTs. So far, surgical resection is the dominant and preferred therapy for IMTs. In addition, steroids, nonsteroidal anti-inflammatory drugs (NSAIDs), chemotherapy, and radiotherapy have been used as adjuvant therapy if necessary ${ }^{[5]}$. Up to now, there are only several studies ${ }^{[3,6-8]}$ of urinary system IMTs relied on enormous population. Unfortunately, these studies described mainly the clinicopathological characteristics so that all of them maybe not illustrate the incidence, survival, mortality and prognosis of urinary system IMTs.

In this study, we are aiming to retrospectively investigate the clinic characteristics, pathological features, treatment, survival and prognosis of urinary system IMTs in China. 


\section{Methods}

We searched pathologic databases at the West China Hospital of Sichuan University and the Third People's Hospital of Chengdu from May 2009 to January 2019. Our search terms included inflammatory myofibroblastic tumor, inflammatory pseudosarcoma, and spindle cell lesion. Finally, 33 patients were included in our study.

Patients characteristics including age, sex, signs and symptoms (including pain, dysuria, urinary frequency, anemia level, tumor size in maximal dimension, tumor location, treatment given, and presence of any tumor recurrence or distant metastases were recognized. Hematuria was defined as red blood cell of urine more than $3 / \mathrm{HP}$.

Available immunohistochemistry $(\mathrm{IHC})$ performed on the diagnostic specimens was reported and staining for ALK, SMA, MSA, Vimentin, Desmin, EMA, CK, BCL-2, Myogenin, CD34, CD21, CD117, Caldesmon, CD10, CD30, CD68, CD99, S100, PCK, and Ki67. Recurrence free survival (RFS) was measured from the time of diagnosis to disease recurrence. Disease free survival (DFS) was defined as the time of diagnosis to disease recurrence or death from any cause. Overall survival (OS) was measured from the time of diagnosis to the time of death from any cause or of last follow-up.

\section{Results}

\section{Baseline characteristics}

Thirty-three patients diagnosed with urogenital system IMTs were identified, which met the eligibility criteria for our study from May 2009 to January 2019. The baseline characteristics are shown in Table 1. A total of 13 patients (39.4\%) were kidney IMTs vs. 10 bladder IMTs (30.3\%) vs. 10 other sites IMTs (30.3\%) which included one urinary tract IMT, one adrenal gland IMT, three testis IMTs, one groin IMT, two pelvis IMTs, and two retroperitoneum IMTs. For all the patients, median age was 39.5 years (range 0-74 years). Median age of kidney IMTs vs. bladder IMTs vs. other sites IMTs was 39 years (range 6-74 years) vs. 51.5 years (range $25-72$ years) vs. 30.5years (0-70 years). Female involvement was slightly more common than male (51.5\% vs. $48.5 \%$ ). Median tumor size of all the patients was $4 \mathrm{~cm}$ (range $1-15 \mathrm{~cm}$ ), and the median tumor size of kidney IMTs was $4 \mathrm{~cm}$ (range 1-15 cm) vs. $4.25 \mathrm{~cm}$ (range 2-7.5 cm) of bladder IMTs vs. $4.4 \mathrm{~cm}$ (range $3-11 \mathrm{~cm}$ ).

The most common presentations were hematuria (13 patients, $39.4 \%)$, pain (12 patients, $36.4 \%$ ), and mass (12 individuals, $36.4 \%$ ) for all the patients. Moreover, the most common clinical symptoms of kidney IMTs were pain (7 patients, $53.8 \%$ ) and mass (6 patients, $46.2 \%$ ) vs. hematuria (7 patients, $70 \%$ ) for bladder IMTs. Thirteen patients with hematuria included three kidney IMTs patients, seven bladder IMTs patients, and three other sites IMTs patients. Twelve individuals with pain were composed of seven kidney IMTs patients, two bladder IMTs patients, three other sites IMTs patients. In addition, twelve patients with mass consisted of six kidney IMTs patients, three bladder IMTs patients, and three other 
sites IMTs. Other clinical symptoms included dysuria (one patients), urinary frequency (two patients), and fever (two patients). What's more, of all patients, anemia was detected in fourteen patients $(42.1 \%)$, including two kidney IMTs patients, seven bladder IMTs, and five other sites IMTs individuals. Ten patients with mild anemia consisted of two kidney IMTs patients, four bladder IMTs patients, and four other sites IMTs. Nevertheless, four patients with moderate anemia included three bladder IMTs patients and one other sites IMTs patient. In general, anemia was recognized in seven patients (70\%) with bladder IMTs.

Significant inflammatory cell types were reported in a total of 16 (48.5\%) patients. Among 16 patients, significant numbers of neutrophils, eosinophils, plasma cells, and lymphocytes were reported in 5 (31.3\%), 8 (50.0\%), 15 (93.8\%), and 8(50.0\%) individuals.

\section{Patients' treatment}

The therapy administrated is summarized in Table 2. In our study, all the patients were treated with surgery. Among patients with kidney IMTs, 5 (38.5\%) patients underwent partial nephrectomy and 8 (61.5\%) patients received radical nephrectomy. Beyond that, among bladder IMTs individuals, most patients (7 patients, $70.0 \%$ ) was administrated with transurethral resection of bladder tumor (TURBT), followed by partial cystectomy ( 5 patients, $50.0 \%$ ), and radical cystectomy ( 1 patients, $10.0 \%$ ). Among those patients who had TURBT performed, specifically, 3 (42.9\%) patients had further partial cystectomy. Therefore, among patients with bladder IMTs, $3(30.0 \%)$ patients were treated with TURBT plus partial cystectomy. In addition, among other sites IMTs patients, 3 (30.0\%) patients underwent orchiectomy, and other patients $(70.0 \%)$ were treated with pure tumor resection. What's more, all the patients didn't undergo additional therapy apart from surgery.

\section{Patients' outcome}

Follow-up was available in 23 patients. After a median follow-up of 78.3 months (range 19.2-118.8 months), the survival data of all the individuals was collected. Interestingly, among thirty-three patients diagnosed with IMTs, only one (3.0\%) patient died from retroperitoneum IMTs at three months after retroperitoneum tumor resection. In addition, among other thirty-two (97.0\%) IMTs patients, none of them developed any local recurrence or distant metastasis upon computed tomography scan and laboratory tests results.

\section{Immunohistochemistry features}

Upon review of the immunohistochemistry results, it was noted that different sets of staining were performed for each patients of our study. The immunohistochemistry results were summarized in Table 3. Anaplastic lymphoma kinase (ALK) was positive in 10 out of $23(43.5 \%)$ patients. Smooth muscle actin (SMA) was positive in 22 out of 25 (88.0\%) patients. Muscle-specific actin (MSA) was positive in 8 out of 
9 (88.9\%) patients. Vimentin was positive in 1 out of 1 (100\%) patients, and CD68 was positive in 3 out of $3(100 \%)$ individuals. Desmin was positive in 13 out of $23(56.5 \%)$ patients. One out of 3 individuals (33.3\%) was positive for cytokeratin (CK). CD34 was positive in 15 out of $17(88.2 \%)$ patients. Both CD117 and CD99 were positive in 1 out of $4(25.0 \%)$ patients. Caldesmon was positive in 2 out of 4 (50.0\%) cases, and CD10 was positive in 1 out of $2(50.0 \%)$ individuals. One out of $18(5.6 \%)$ patients was positive for S100. PCK was positive in 3 out of 11 (27.3\%) cases. What's more, none of the 33 individuals were positive for epithelial membrane antigen (EMA) (0/13), Bcl-2 (0/3), Myogenin (0/5), CD21 (0/3), CD30 (0/1). The expression of Ki67 was detected in 15 out of 33 (45.5\%) patients. Median of Ki67 expression in IMTs was $2 \%$ (range 1\%-25\%). Last but not least, among 3 patients performed fluorescence in situ hybridization (FISH) analysis, a total of $2(67.7 \%)$ patients showed a positive $A L K$ translocation.

\section{Discussion}

IMTs, a rare subtype with unique clinical and pathologic characteristics, in which the dominant components is spindle cells, and other components including alterable extracellular collagen, lymphocytes, and plasma cells ${ }^{[9]}$. IMTs, originally reported in the lung, has been recognized at multiple extrapulmonary sites, particularly the soft tissue and solid organs of children and young adults ${ }^{[1,3,8]}$. The the most common site of IMTs of genitourinary system is the urinary bladder. The alternative terminologies of IMTs, including plasma cell granuloma, inflammatory pseudotumor, pseudosarcomatous fibromyxoid tumor, and inflammatory myofibroblastic tumor, demonstrated that further studies are needed to explore the pathogenesis of IMTs. Nevertheless, a crowd of studies, including our study, agree with the myofibroblastic nature of IMTs with expression of SMA, desmin, vimentin, CD34, and CD68. At the same time, we found that the significant inflammatory cell types of IMTs included neutrophil, eosinophil, plasma cell, and lymphocyte, which supported the prior reports ${ }^{[10]}$. According to the current WHO Classification of Tumors of Soft tissue and Bone ${ }^{[11]}$, moreover, IMTs are categorized as intermediate grade tumors in view of the potential for local recurrence and distant metastasis.

The first case report of IMTs of the kidney was published in 1976, closely followed by a series of case reports and rare studies ${ }^{[9,12-16]}$. The median age of kidney IMTs 39 years (range 6-74 years) in our study doesn't consist with the mean age 57.1 years in a large study ${ }^{[9]}$, but consists with the mean age 43.3 years of literatures review in the 13 case reports. In addition, it agrees with other reports that the age of onset, concentrated in 25-72 years (median 51.5 years), and the most common symptom is hematuria with more than half of them being anemia upon presentation of bladder IMTs in our study $[6,8,17]$. Sarcomatoid urothelial carcinoma et al are the most significant subtypes in the differential diagnosis of IMTs. What's more, both IMTs and sarcomatoid urothelial carcinoma exhibit similar biological characteristics resulting in the differential diagnosis becoming the most difficult ${ }^{[6]}$. According to the study of Liang et al ${ }^{[18]}$, however, the good news is that contrast-enhanced CT may be valuable in the diagnostic imaging of the urinary system IMTs. 
The ALK gene translocation was detected by FISH in 2/3 (67.7\%) individuals, which is consistent with $30 \%-67 \%$ ALK gene rearrangements of IMTs on FISH testing in other studies $[6,17,19,20]$. As is well-known, more than half of anaplastic large cell lymphoma (ALCL) patients presented ALK gene translocation accompanied with tyrosine kinase activation. Moreover, ALK gene rearrangement was confirmed in testis IMTs too ${ }^{[21]}$. Therefore, IMTs were considered as neoplastic rather than reactive in mature on the basis of ALK gene rearrangement. In 2014, Chun et al ${ }^{[10]}$ published a systematic review in which they demonstrated that had a marked female predilection (male: female=1: 1.67) was recognized in ALKpositive IMTs.

Nowadays, surgical resection is the dominant therapy of urogenital system IMTs. In 2003, a 12 kidney IMTs cases study was published in which all the patients underwent kidney resection, was administrated with no adjuvant treatment, and no recurrent tumors was recognized in the patients available for followup ${ }^{[9]}$. Our study happened to coincide with this series ${ }^{[9]}$ on kidney IMTs patients' prognosis.

In terms of the urinary bladder IMTs, according to previous reports, partial or radical cystectomy can insure no residual IMTs ${ }^{[10]}$. In consideration of benign behavior of the urinary bladder IMTs, nevertheless, TURBT maintained as a selection in reserve for patients who are unwilling to received partial or radical surgery. Studies including our series demonstrated that local management can make patients acquire favorable recurrence free survival and overall survival ${ }^{[3,6,17]}$. Until now, although extremely rare recurrence was reported in fewer individuals, no distant metastasis was illustrated in literatures already. In our study, one patient with retroperitoneum IMTs appeared poor prognosis after retroperitoneum tumor resection. In addition, a systematic review published in 2014 also demonstrated local tumor recurrence in five bladder IMTs cases ${ }^{[10]}$. Therefore, although further chemotherapy or radiotherapy is not needed, close follow-up, regular cystoscopy and imaging tests are necessary for the early diagnosis of tumor recurrence and metastasis.

\section{Conclusion}

This study confirmed that urogenital system IMTs, with potential of recurrence or metastasis, are extremely rare. In summary, although favorable prognosis was observed in IMTs of urogenital system, closely follow-up is essential and needed.

\section{Abbreviations}

IMTs=Inflammatory myofibroblastic tumors;

$\mathrm{IHC}=$ immunohistochemistry;

RFS=Recurrence free survival;

DFS=Disease free survival; 
OS=Overall survival;

TURBT=transurethral resection of bladder tumor;

$\mathrm{FISH=fluorescence} \mathrm{in} \mathrm{situ} \mathrm{hybridization;}$

ALK=anaplastic lymphoma kinase;

$\mathrm{SMA}=$ smooth muscle actin;

$M S A=$ muscle-specific actin;

$E M A=e p i t h e l i a l$ membrane antigen;

CK=cytokeratin;

\section{Declarations}

\section{Ethics approval and consent to participate}

The research was based on a retrospective study. Ethics approval was not needed because the study is a secondary analysis of an existing public internal database of our hospital.

\section{Consent for publication}

Not applicable

\section{Availability of data and materials}

All data on which the conclusions of the manuscript rely are available from reasonable request.

\section{Competing interests}

All the authors declared no competing interests.

\section{Funding}

This research was funded by the National Natural Science Foundation of China (Grant no. 81370855区 81770857 and 81200551) and Project of Sichuan Province Science and Technology Department (Grant no. 2015SZ0230 and 2017KJT0034). Data collection, analysis and publication charges are funded by the projects. The funders of the projects conceived and designed the work.

\section{Authors' contributions}

$\mathrm{BC}$ and $\mathrm{HX}$ designed the study, collected and drafted the manuscript. DC, JG, ZC participated in the design of the study, performed the procedures. SQ and TL performed the economic and statistical analysis. NM and YH participated in the study design and coordination. $\mathrm{LL}$ and QW conceived of the 
study, participated in its design and coordination, supervised the draft. All authors read and approved the final manuscript.

\section{Acknowledgments}

None

\section{References}

[1] Cerier E, Beal EW, Dillhoff ME. Inflammatory myofibroblastic tumour: an unusual presentation including small bowel obstruction and palpable abdominal mass. BMJ Case Rep. 2018. 2018.

[2] Roth JA. Reactive pseudosarcomatous response in urinary bladder. Urology. 1980. 16(6): 635-7.

[3] Coffin CM, Watterson J, Priest JR, Dehner LP. Extrapulmonary inflammatory myofibroblastic tumor (inflammatory pseudotumor). A clinicopathologic and immunohistochemical study of 84 cases. Am J Surg Pathol. 1995. 19(8): 859-72.

[4] Rotenberry C, Dowd K, Russell D, DeRiese W, Teeple S, Cammack T. Robot-assisted Partial Cystectomy for Treatment of Inflammatory Myofibroblastic Tumor of the Bladder. Urol Case Rep. 2017. 11: 25-27.

[5] Tao YL, Wang ZJ, Han JG, Wei P. Inflammatory myofibroblastic tumor successfully treated with chemotherapy and nonsteroidals: a case report. World J Gastroenterol. 2012. 18(47): 7100-3.

[6] Montgomery EA, Shuster DD, Burkart AL, et al. Inflammatory myofibroblastic tumors of the urinary tract: a clinicopathologic study of 46 cases, including a malignant example inflammatory fibrosarcoma and a subset associated with high-grade urothelial carcinoma. Am J Surg Pathol. 2006. 30(12): 1502-12.

[7] Telugu RB, Prabhu AJ, Kalappurayil NB, Mathai J, Gnanamuthu BR, Manipadam MT.

Clinicopathological Study of 18 Cases of Inflammatory Myofibroblastic Tumors with Reference to ALK-1 Expression: 5-Year Experience in a Tertiary Care Center. J Pathol Transl Med. 2017. 51(3): 255-263.

[8] Teoh JY, Chan NH, Mak SM, et al. Inflammatory myofibroblastic tumours of the urinary bladder: multicentre 18-year experience. Urol Int. 2015. 94(1): 31-6.

[9] Kapusta LR, Weiss MA, Ramsay J, Lopez-Beltran A, Srigley JR. Inflammatory myofibroblastic tumors of the kidney: a clinicopathologic and immunohistochemical study of 12 cases. Am J Surg Pathol. 2003. 27(5): 658-66.

[10] Teoh JY, Chan NH, Cheung HY, Hou SS, Ng CF. Inflammatory myofibroblastic tumors of the urinary bladder: a systematic review. Urology. 2014. 84(3): 503-8.

[11] Rosenberg AE. WHO Classification of Soft Tissue and Bone, fourth edition: summary and commentary. Curr Opin Oncol. 2013. 25(5): 571-3. 
[12] Tazi K, Ehirchiou A, Karmouni T, et al. [Inflammatory pseudotumors of the kidney: a case report]. Ann Urol (Paris). 2001. 35(1): 30-3.

[13] Ishikawa T, Fujisawa M, Tamada H, Inoue T, Shimatani N. Inflammatory pseudotumor of the kidney. Int J Urol. 2004. 11(5): 337-9.

[14] Bildirici K, Dönmez T, Gürlek E. Inflammatory pseudotumor of the kidney. Int Urol Nephrol. 2004. 36(2): 141-3.

[15] Lee NG, Alexander MP, Xu H, Wang DS. Renal Inflammatory Myofibroblastic Tumor: A Case Report and Comprehensive Review of Literature. World J Oncol. 2011. 2(2): 85-88.

[16] Jenkins LC, Whittington E, Ciancio G, Jorda M. Inflammatory myofibroblastic tumor of the kidney. J Urol. 2013. 189(6): 2310-1.

[17] Harik LR, Merino C, Coindre JM, Amin MB, Pedeutour F, Weiss SW. Pseudosarcomatous myofibroblastic proliferations of the bladder: a clinicopathologic study of 42 cases. Am J Surg Pathol. 2006. 30(7): 787-94.

[18] Liang W, Zhou X, Xu S, Lin S. CT Manifestations of Inflammatory Myofibroblastic Tumors (Inflammatory Pseudotumors) of the Urinary System. AJR Am J Roentgenol. 2016. 206(6): 1149-55.

[19] Cessna MH, Zhou H, Sanger WG, et al. Expression of ALK1 and p80 in inflammatory myofibroblastic tumor and its mesenchymal mimics: a study of 135 cases. Mod Pathol. 2002. 15(9): 931-8.

[20] Freeman A, Geddes N, Munson P, et al. Anaplastic lymphoma kinase (ALK 1) staining and molecular analysis in inflammatory myofibroblastic tumours of the bladder: a preliminary clinicopathological study of nine cases and review of the literature. Mod Pathol. 2004. 17(7): 765-71.

[21] Hickman RA, Alexander M, Provet J, Melamed J, Deng FM. Inflammatory myofibroblastic tumour of the testis with confirmed anaplastic lymphoma kinase gene rearrangement. Histopathology. 2016. 68(7): 1109-11.

\section{Tables}

Table1. Clinical characteristics of patients diagnosed with IMTs. 


\begin{tabular}{|c|c|c|c|c|}
\hline Characteristics & $\operatorname{Total}(\mathrm{n}=33) \%$ & Kidney $(\mathrm{n}=13) \%$ & Bladder $(\mathrm{n}=10) \%$ & $\begin{array}{c}\text { Other } \\
\text { sites* } \\
(\mathrm{n}=10) \%\end{array}$ \\
\hline \multicolumn{5}{|l|}{ Age(years) } \\
\hline Median & 39.5 & $\begin{array}{c}39 \\
6-71\end{array}$ & $\begin{array}{l}51.5 \\
25.72\end{array}$ & 30.5 \\
\hline \multicolumn{5}{|l|}{ Sex } \\
\hline $\begin{array}{l}\text { Female } \\
\text { Male }\end{array}$ & $\begin{array}{l}17(51.5) \\
16(48.5)\end{array}$ & $\begin{array}{l}5(38.5) \\
8(61.5)\end{array}$ & $\begin{array}{l}7(70.0) \\
3(30.0)\end{array}$ & $\begin{array}{l}5(50.0) \\
5(50.0)\end{array}$ \\
\hline \multicolumn{5}{|l|}{ Tumor size $(\mathrm{cm})$} \\
\hline $\begin{array}{l}\text { Median } \\
\text { Range }\end{array}$ & $\begin{array}{c}4 \\
1-15\end{array}$ & $\begin{array}{c}4 \\
1-15\end{array}$ & $\begin{array}{l}4.25 \\
2-7.5\end{array}$ & $\begin{array}{c}4.4 \\
3-11\end{array}$ \\
\hline \multicolumn{5}{|l|}{ Clinical presentation } \\
\hline Hematuria & $13(39.4)$ & $3(23.1)$ & $7(70.0)$ & $3(30.0)$ \\
\hline $\begin{array}{l}\text { Dysuria } \\
\text { Urinary frequency }\end{array}$ & $\begin{array}{l}1(3.0) \\
2(6.1)\end{array}$ & $\begin{array}{l}0 \\
0\end{array}$ & $\begin{array}{c}0 \\
2(20.0)\end{array}$ & $1(10.0)$ \\
\hline Pain & $12(36.4)$ & $7(53.8)$ & $2(20.0)$ & $3(30.0)$ \\
\hline Fever & $2(6.1)$ & $2(15.4)$ & 0 & \\
\hline Mass & $12(36.4)$ & $6(46.2)$ & $3(30.0)$ & $3(30.0)$ \\
\hline $\begin{array}{l}\text { Anemra } \\
\text { Mild }\end{array}$ & $10(30.0)$ & $2(15.4)$ & $4(40.0)$ & $4(40.0)$ \\
\hline Moderate & $4(12.1)$ & 0 & $3(30.0)$ & $1(10.0)$ \\
\hline \multicolumn{5}{|l|}{ Inflammatory cell types\# } \\
\hline Neutrophils & $5 / 16(31.3 \%)$ & & & \\
\hline Eosinophils & $8 / 16(50.0 \%)$ & & & \\
\hline Plasma cells & 15/16(93.8\%) & & & \\
\hline Lymphocytes & $8 / 16(50.0 \%)$ & & & \\
\hline
\end{tabular}

* Other sites include urinary tract, adrenal gland, testis, pelvis, retroperitoneum, and groin. \# Inflammatory cell types, among 33 cases, only 16 patients' exact inflammatory cell types was reported.

Table2. Patients' treatment

\begin{tabular}{llll}
\hline Tumor entity & & Treatment administrated & No. (\%) \\
\hline Kidney IMTs $(\mathrm{n}=13)$ & & Partial nephrectomy & $5(38.5)$ \\
& & Radical nephrectomy & $8(61.5)$ \\
\hline Bladder IMTs $(\mathrm{n}=10)$ & & TURBT & $4(40.0)$ \\
& & TURBT + partial cystectomy & $3(30.0)$ \\
& & Partial cystectomy & $2(20.0)$ \\
\hline Other sites IMTs(n=10) & Urinary tract $(\mathrm{n}=1)$ & Radical cystectomy & $1(10.0)$ \\
\cline { 2 - 4 } & Adrenal gland $(\mathrm{n}=1)$ & Tumor resection & $1(10.0)$ \\
& Testis $(\mathrm{n}=3)$ & Orchiectomy & $1(10.0)$ \\
& Pelvis $(\mathrm{n}=2)$ & Tumor resection & $3(30.0)$ \\
& Retroperitoneum(n=2) & Tumor resection & $2(20.0)$ \\
& Groin $(\mathrm{n}=1)$ & Tumor resection & $2(20.0)$ \\
& & & $1(10.0)$ \\
\hline
\end{tabular}

IMTs, inflammatory myofibroblastic tumors; TURBT, transurethral resection of bladder tumor 
Table3. Results of immunohistochemistry staining of all patients with IMTs

\begin{tabular}{lcc}
\hline Markers(No. of tests) & Positive (No. and \%) & Negative (No. and \%) \\
\hline ALK(n=23) & $10(43.5)$ & $13(56.5)$ \\
SMA(n=25) & $22(88.0)$ & $3(12.0)$ \\
MSA(n=9) & $8(88.9)$ & $1(11.1)$ \\
Vimentin(n=1) & $1(100)$ & 0 \\
Desmin $(n=23)$ & $13(56.5)$ & $10(43.5)$ \\
EMA(n=13) & 0 & $13(100)$ \\
CK(n=3) & $1(33.3)$ & $2(66.7)$ \\
BCL-2 $(n=3)$ & 0 & $3(100)$ \\
Myogenin(n=5) & 0 & $5(100)$ \\
CD34 $(n=17)$ & $15(88.2)$ & $2(11.8)$ \\
CD21 $(n=3)$ & 0 & $3(100)$ \\
CD117(n=4) & $1(25.0)$ & $3(75.0)$ \\
Caldesmon $(n=4)$ & $2(50.0)$ & $2(50.0)$ \\
CD10(n=2) & $1(50.0)$ & $1(50.0)$ \\
CD30 $(n=1)$ & 0 & $1(100)$ \\
CD68 $(n=3)$ & $3(100)$ & 0 \\
CD99 $(n=4)$ & $1(25.0)$ & $3(75.0)$ \\
S100 $(n=18)$ & $1(5.6)$ & $17(94.4)$ \\
PCK $(n=11)$ & $3(27.3)$ & $8(72.7)$ \\
\hline
\end{tabular}

IMTs, inflammatory myofibroblastic tumors; ALK, anaplastic lymphoma kinase; SMA, smooth muscle actin; MSA, muscle-specific actin; EMA, epithelial membrane antigen; CK, cytokeratin. 